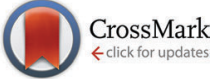

Cite this: Phys. Chem. Chem. Phys., 2016, 18, 331

Received 19th November 2015, Accepted 23rd November 2015

DOI: $10.1039 / c 5 c p 07117 h$

www.rsc.org/pccp

\section{Can ferroelectric polarization explain the high performance of hybrid halide perovskite solar cells? $\uparrow$}

\author{
Tejas S. Sherkar* and L. Jan Anton Koster
}

\begin{abstract}
The power conversion efficiency of photovoltaic cells based on the use of hybrid halide perovskites, $\mathrm{CH}_{3} \mathrm{NH}_{3} \mathrm{PbX}_{3}(\mathrm{X}=\mathrm{Cl}, \mathrm{Br}, \mathrm{I})$, now exceeds $20 \%$. Recently, it was suggested that this high performance originates from the presence of ferroelectricity in the perovskite, which is hypothesized to lower charge recombination in the device. Here, we investigate and quantify the influence of mesoscale ferroelectric polarization on the device performance of perovskite solar cells. We implement a 3D drift diffusion model to describe the solar cell operation. To account for the mesoscale ferroelectricity, we incorporate domains defined by polarization strength, $P$, in 3D space, forming different polarization landscapes or microstructures. Study of microstructures with highly-ordered polarized domains shows that charge transport and recombination in the solar cell depends significantly on the polarization landscape viz. the orientation of domain boundaries and the size of domains. In the case of the microstructure with random correlated polarization, a realistic scenario, we find indication of the existence of channels for efficient charge transport in the device which leads to lowering of charge recombination, as evidenced by the high fill factor (FF). However, the high open-circuit voltage $\left(V_{O C}\right)$, which is typical of high performance perovskite solar cells, is unlikely to be explained by the presence of ferroelectric polarization in the perovskite.
\end{abstract}

\section{Introduction}

Photovoltaic cells based on the use of hybrid halide perovskites, $\mathrm{CH}_{3} \mathrm{NH}_{3} \mathrm{PbX}_{3}(\mathrm{X}=\mathrm{Cl}, \mathrm{Br}, \mathrm{I})$, have recently attracted great interest. ${ }^{1-4}$ These materials show desirable properties for use in solar cells, such as, favorable direct band gap, large absorption coefficient, high ambipolar charge mobilities, and long charge diffusion lengths. ${ }^{5-7}$ As a result, there has been a dramatic rise in their power conversion efficiency from $3.8 \%$ in $2009,{ }^{5}$ to $19 \%$ in $2015,{ }^{8}$ and a certified value of $20.1 \%$. ${ }^{9}$ This excellent performance also derives from the finding that, in perovskite solar cells (PSC), the recombination of charge carriers is significantly low. ${ }^{10}$ Ferroelectricity has been hypothesized to play a key role in reducing charge recombination, ${ }^{11,12}$ and therefore is of considerable interest for further investigation.

In the context of photovoltaics, ferroelectricity has been extensively studied in inorganic materials, including inorganic perovskites. ${ }^{13-19}$ A number of effects have been attributed to the ferroelectric nature of these materials such as the above

Zernike Institute for Advanced Materials, University of Groningen, Nijenborgh 4, 9747AG, Groningen, The Netherlands. E-mail: t.sherkar@rug.nl

$\dagger$ Electronic supplementary information (ESI) available: Additional simulation results. See DOI: $10.1039 / \mathrm{c} 5 \mathrm{cp} 07117 \mathrm{~h}$ band gap voltages and their switchable behavior. ${ }^{17,18,20}$ In these materials, the photovoltaic effect arises from charge separation at ferroelectric domain boundaries to generate a photocurrent. ${ }^{18,19}$ Recently, ferroelectricity has also been observed experimentally in hybrid halide perovskites, where sizeable $(1100 \mathrm{~nm})$ ferroelectric domains were shown to exist in $\mathrm{CH}_{3} \mathrm{NH}_{3} \mathrm{PbI}_{3}$ films. ${ }^{21}$ These ferroelectric domains are typically characterized by polarization resulting from the tetragonal $\mathrm{PbX}_{6}$ cage, halide ion off-centering and the methylammonium $\left(\mathrm{MA}=\mathrm{CH}_{3} \mathrm{NH}_{3}\right)$ cation orientation. ${ }^{22,23}$ Theoretical calculations showed that the orientation of molecular cations gives rise to an electrostatic landscape which can form internal pathways for efficient charge separation and transport in hybrid perovskites. ${ }^{11,24}$ On a mesoscale, it was suggested that the domain boundaries in hybrid perovskites can act as channels for charge transport. ${ }^{12}$ While, Frost and Liu et al. provide a key insight into the properties of hybrid halide perovskites, ${ }^{11,12}$ a quantitative influence of ferroelectricity on the device performance of PSCs is still lacking.

The origin of the low charge recombination found in PSCs can be said to be due to: (a) the excellent material properties, such as the high carrier mobilities and their long diffusion lengths, which then translates into an intrinsically low recombination strength of the perovskite, or (b) the creation of channels for efficient carrier transport resulting from ferroelectric polarization 
in perovskite, which then lowers the apparent recombination strength of the perovskite, or (c) a combination of both. In this context, it would therefore be of great interest to study the effect of ferroelectricity on the device characteristics of PSCs and clarify its role in the lowering of the apparent charge recombination strength.

In this paper, we report the influence of mesoscale ferroelectric polarization on the device performance of PSCs. We implement a three dimensional (3D) drift diffusion model to describe charge generation, transport and recombination in the device. The model also takes into account the mesoscale ferroelectricity by establishing domains defined by the polarization, $P$, in 3D space. Initially, we study two highly-ordered microstructures, with domain boundaries parallel and perpendicular to the electrodes respectively. Next, we study the realistic scenario of a microstructure with random correlated polarization, which can be envisaged as a composite of the two highly-ordered microstructures. To quantify the contribution of the ferroelectric polarization to the device performance in the context of charge recombination, we investigate the following cases: (a) high recombination strength plus the ferroelectric polarization and, (b) low recombination strength.

We show that the device performance depends significantly on the domain boundary orientation and the domain size. Ordered domains parallel to the electrodes and having net polarization direction along the thickness of the device, results in poor device performance as electrons and holes buildup at the domain boundaries and eventually recombine to give low fill factor (FF), with marginal improvement in open-circuit voltage $\left(V_{\text {OC }}\right)$. A device with ordered domains perpendicular to the electrodes and having net polarization direction in the plane of the device shows high short-circuit current $\left(J_{\mathrm{SC}}\right)$ and $\mathrm{FF}$, as electrons and holes encounter no domain boundaries during their transport toward the electrodes. In the case of random correlated polarization in domains, efficient transport of electrons and holes and their low recombination in the device gives rise to high FFs, which indicates the existence of channels for charge transport. However, a high $V_{\text {OC }}$ (relative to the band gap of the perovskite), which is a distinctive feature of PSCs, ${ }^{25}$ cannot be explained by mesoscale ferroelectric polarization. Using a simple model to describe the recombination in PSCs in a bimolecular way, we are able to show that, only the consideration of reduction in bimolecular recombination strength is able to successfully describe the high $V_{\text {OC }}$ of high performance PSCs.

\section{Results and discussion}

\section{Ferroelectric polarization}

Ferroelectric materials are characterized by domains that are formed by the distribution of spontaneous polarization in space. A typical ferroelectric material with domains is shown in Fig. 1. The component of the polarization perpendicular to the domain boundary gives rise to bound charge at the boundary following ${ }^{26}$

$$
\rho_{\text {pol }}=-\nabla \cdot \mathbf{P}
$$

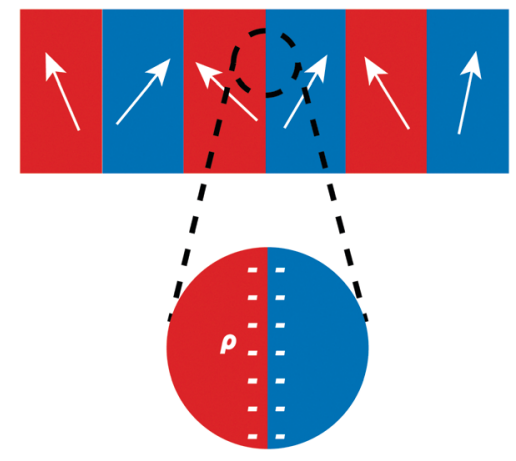

Fig. 1 Domains created by spontaneous polarization in a ferroelectric material. Arrows indicate the direction of net polarization in domains. The component of the polarization vector perpendicular to the domain boundary results in a bound charge density, $\rho$, on the boundary.

where $\rho_{\text {pol }}$ is the bound charge density. This bound charge leads to a depolarizing electric field in the direction opposite to the spontaneous polarization. The depolarizing field can then influence the dynamics of photo-induced charges as in a perovskite solar cell. Therefore, the solar cell performance would depend upon the combined effect of the depolarizing field in domains and the external electric field (bias). The treatment of the bound charge in the simulation to account for the ferroelectric nature of the perovskite is detailed in the next section.

\section{D drift-diffusion model}

The structure of the planar perovskite solar cell used in the device simulation is as shown in Fig. 2. The crystalline structure of the perovskite is associated with well-defined valence and conduction bands separated by a band gap of $1.5 \mathrm{eV}$.

In a typical planar perovskite solar cell with a $\mathrm{p}-\mathrm{i}-\mathrm{n}$ structure, the interface recombination at the doped hole (p) and electron (n) transport layer is found to be primarily dependent on the interface trap density and the energy level offsets, ${ }^{30-33}$ whereas the recombination in the perovskite absorber (i) layer is an intrinsic material property. Therefore, to exclusively study the charge recombination in the absorber layer, where the ferroelectric polarization is dominant, the $\mathrm{p}$ and $\mathrm{n}$ layers are not taken into account in the model. In addition, to disregard

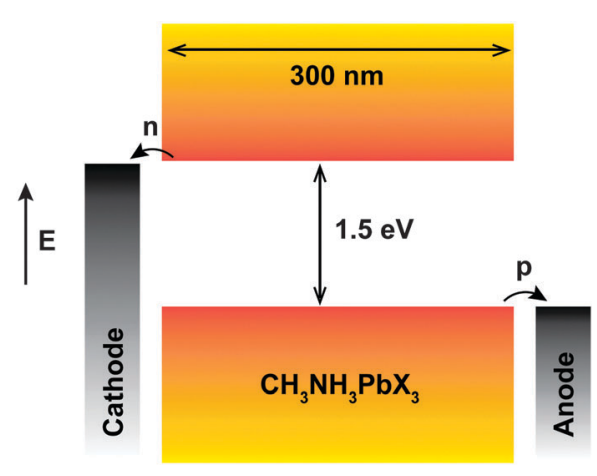

Fig. 2 Device structure of the perovskite solar cell used in simulation. The energy offsets at both electrodes are $0.0 \mathrm{eV}$. 
the role of any interface energy loss on the device performance, the valence band offset and the conduction band offset between anode/perovskite and perovskite/cathode, respectively, are set to $0.0 \mathrm{eV}$ as seen in Fig. 2. Device structures without a transport layer were recently also studied experimentally, and shown to achieve high efficiencies including high open-circuit voltage. ${ }^{34-36}$

Our formulation of the 3D model is based on the drift-diffusion equations for electrons and holes throughout the device and on solving the Poisson's equation in three dimensions. ${ }^{37}$ In the perovskite layer, the absorption of light is shown to create both excitons and free charges. ${ }^{38,39}$ However, owing to the low exciton binding energy $(22 \mathrm{meV}),{ }^{38}$ we assume the generation of free charges throughout the perovskite absorber. The transport of these free charges is then governed by diffusion and electrically induced drift; for electrons ${ }^{37}$

$$
J_{\mathrm{n}}=-q n \mu_{\mathrm{n}} \nabla V+q D_{\mathrm{n}} \nabla n
$$

and for holes

$$
J_{\mathrm{p}}=-q p \mu_{\mathrm{p}} \nabla V-q D_{\mathrm{p}} \nabla p .
$$

where $q$ is the electronic charge $\left(1.602 \times 10^{-19} \mathrm{C}\right), V$ is the electrostatic potential, $n$ and $p$ are electron and hole concentrations, $\mu_{\mathrm{n}}$ and $\mu_{\mathrm{p}}$ are electron and hole mobilities and, $D_{\mathrm{n}}$ and $D_{\mathrm{p}}$ are electron and hole diffusion constants respectively.

The electrostatic potential is solved from the Poisson's equation

$$
\nabla^{2} V=-\left(\frac{q}{\varepsilon}(p-n)+\rho_{\mathrm{pol}}\right)
$$

where $\varepsilon$ is the permittivity of the perovskite and $\rho_{\text {pol }}$ is the bound charge density given by eqn (1). The inclusion of $\rho_{\mathrm{pol}}$ in the Poisson's equation makes it possible to account for the role of mesoscale ferroelectricity in the perovskite. The boundary condition on the electrostatic potential is

$$
q\left(V_{\mathrm{L}}-V_{\mathrm{R}}+V_{\text {applied }}\right)=W_{\text {anode }}-W_{\text {cathode }}
$$

where $V_{\mathrm{L}}$ and $V_{\mathrm{R}}$ are potentials at either electrodes, $V_{\text {applied }}$ is the applied voltage, and $W_{\text {anode }}$ and $W_{\text {cathode }}$ are the anode and cathode work functions, respectively.

The electron (hole) density at the electrodes, in case of zero energy offset between the LUMO (HOMO) of the perovskite and the electrodes is given by the density of states, $N_{\mathrm{c} / \mathrm{v}}$, for the perovskite. Given the effective mass of charge carriers in the perovskite, $m^{*}$, the density of states is calculated from the relation ${ }^{40}$

$$
N_{\mathrm{c} / \mathrm{v}}=2\left(2 \pi m^{*} k T / h^{2}\right)^{\frac{3}{2}}
$$

Charge carriers in $\mathrm{CH}_{3} \mathrm{NH}_{3} \mathrm{PbX}_{3}$ can recombine via both, bimolecular and single carrier trapping (Shockley-Read-Hall) mechanism. However, being a direct band gap semiconductor, 5,41 bimolecular recombination is found to be the dominant recombination process. ${ }^{42}$ In addition, experimental works have shown the density of traps to be low, ${ }^{43,44}$ suggesting minor impact on the solar cell performance. In our analysis, we model the charge recombination in the perovskite absorber in a bimolecular way, given by

$$
R=\gamma n p
$$

where $\gamma$ is the recombination rate constant. Owing to the systematic comparison found in the literature between the experimental bimolecular recombination rate in perovskite and the upper limit for bimolecular recombination rate given by the Langevin theory, ${ }^{10}$ we express the recombination rate constant $\gamma$ by the modified Langevin expression ${ }^{45}$

$$
\gamma=\gamma_{\text {pre }} \frac{q}{\varepsilon}\left(\mu_{\mathrm{n}}+\mu_{\mathrm{p}}\right)
$$

where $q$ is the elementary charge, $\varepsilon$ is the dielectric permittivity of the perovskite, and $\gamma_{\text {pre }}$ is the pre-factor that quantifies the reduction in Langevin type bimolecular recombination.

\section{Device parameters}

The parameter estimates for the device used in our simulation are shown in Table 1. Our calculations are based on a cell consisting of lead tri-halide perovskite $\left(\mathrm{CH}_{3} \mathrm{NH}_{3} \mathrm{PbX}_{3}\right)$ as an absorber. The thickness of the perovskite absorber layer is assumed to be $300 \mathrm{~nm}$ which is typical for high performance perovskite solar cells. $^{27}$ The generation of free charges, $G=5 \times 10^{27} \mathrm{~m}^{-3} \mathrm{~s}^{-1}$ is assumed to be uniform throughout the absorber layer. Assuming the effective mass of carriers, $m^{*} \sim 0.3 m_{0},{ }^{46,47}$ the density of states of the valence and the conduction bands of the perovskite is calculated from eqn (6) to be $N_{\mathrm{c} / \mathrm{v}}=4.8 \times 10^{24} \mathrm{~m}^{-3}$.

The polarization in the perovskite is induced by the spontaneous feature i.e. the rotating methylammonium ions (MA) and the permanent $\mathrm{PbX}_{3}$ cage. Theoretical studies have estimated the value of the polarization density for the ferroelectric phase at room temperature to be a few $\mu \mathrm{C} \mathrm{cm}^{-2} \cdot{ }^{23,48}$ To examine the effect due to the permanent polarization on the photovoltaic working mechanism of perovksite solar cell, we initially perform calculations by considering a small polarization value of $0.05 \mu \mathrm{C} \mathrm{cm}{ }^{-2}$ for the highly-ordered microstructures. For the realistic scenario of a microstructure with random correlated polarization, we then perform a study as a function of increasing value of polarization density.

We keep the pre-factor $\left(\gamma_{\text {pre }}\right)$ equal to unity, which signifies high recombination strength, and then simulate the device with and without the presence of ferroelectric polarization in domains. In addition, we vary the size of domains from $15 \mathrm{~nm}$ to $100 \mathrm{~nm}$. Finally, we simulate the device with low recombination strength $\left(\gamma_{\text {pre }}=10^{-4}\right)$ as reported via experiments in the literature. ${ }^{10}$ A study as a function of the mobility and the langevin recombination pre-factor $\left(\gamma_{\text {pre }}\right)$ is presented as additional simulation results in the ESI. $\dagger$

Table 1 Parameter estimates used in device simulation. Here, $\varepsilon_{0}$ is the free space permittivity $\left(\mathrm{F} \mathrm{m}^{-1}\right)$

\begin{tabular}{llll}
\hline Parameter & Symbol & Value & Ref. \\
\hline Perovskite thickness & $L$ & $300 \mathrm{~nm}$ & 27 \\
Perovskite permittivity & $\varepsilon$ & $6.5 \varepsilon_{0}$ & 28 \\
Energy of conduction band in perovskite & $\Phi_{\mathrm{n}}$ & $-3.9 \mathrm{eV}$ & 29 \\
Energy of valence band in perovskite & $\Phi_{\mathrm{p}}$ & $-5.4 \mathrm{eV}$ & 29 \\
Hole mobility in perovskite & $\mu_{\mathrm{p}}$ & $2 \mathrm{~cm}^{2} \mathrm{~V}^{-1} \mathrm{~s}^{-1}$ & 6 \\
Electron mobility in perovskite & $\mu_{\mathrm{n}}$ & $2 \mathrm{~cm}^{2} \mathrm{~V}^{-1} \mathrm{~s}^{-1}$ & 6
\end{tabular}




\section{Influence of ferroelectric polarization on device performance}

The various device microstructures considered in our calculations are shown in Fig. 3a-e. These structures differ in the polarization direction and in domain sizes. Fig. 3 a shows the structure where no polarization exists in the perovskite (from here on, this structure is referred to as the reference structure). Fig. $3 \mathrm{~b}$ and c show structures with polarization in the $\pm X$ and $\pm Y$ directions respectively (from here on, devices with these structures are referred to as vertical and lateral devices respectively). The approach used to obtain the short range (Fig. 3d) and long range (Fig. 3e) correlated structures is mentioned in the Methods section. Fig. 3d and e mimic the domain structures calculated by Frost et al. via the implementation of Monte-Carlo simulation of the polarized domain behavior in hybrid perovskites. ${ }^{24}$

Given any structure, the bound charge is calculated on all domain boundaries (i.e. points where discontinuity in polarization occurs) following eqn (1) and is then incorporated in eqn (4) to be used in 3D drift diffusion simulation. For the high recombination strength $\left(\gamma_{\text {pre }}=1\right)$ scenario, the comparison between the simulated $J-V$ characteristics of the perovskite solar cell for the model microstructures is shown in Fig. 4. As evidenced by the shortcircuit current density $\left(\mathrm{J}_{\mathrm{SC}}\right)$ and fill factor $(\mathrm{FF})$ values, the presence of ferroelectric polarization in domains significantly affects the macroscopic device performance. In all cases, the depolarizing electric field arising from the bound charges at domain boundaries results in accumulation of carriers at domain boundaries as

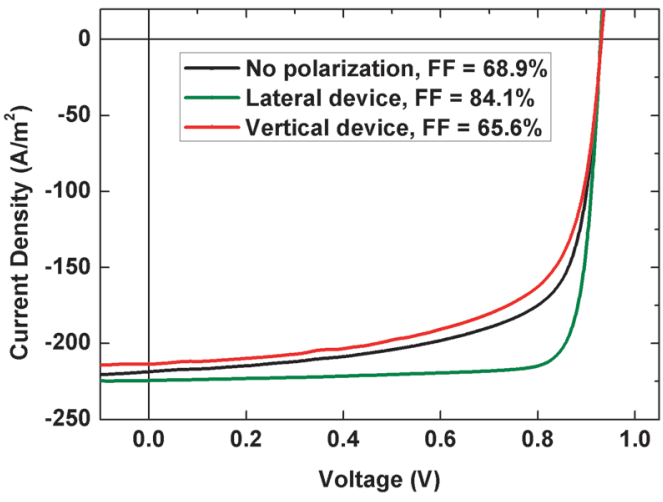

Fig. 4 Simulated J-V curves for device with no polarization (Fig. 3a), vertical device (Fig. 3b) and lateral device (Fig. 3c).

seen from the carrier concentration profiles shown in Fig. 5. The domain boundaries are thus able to effectively separate the charge carriers in the bulk of the material. However, the mere presence of domain boundaries does not necessarily lead to efficient transport and extraction of carriers as explained below.

The potential along the length of the device is mapped in Fig. 6 for the vertical and lateral structures. In the vertical device structure, the domain boundaries act as barriers for efficient carrier transport toward the electrodes. This results in extraction of fewer charge carriers (majority of which are between the electrodes and the first and the last domain

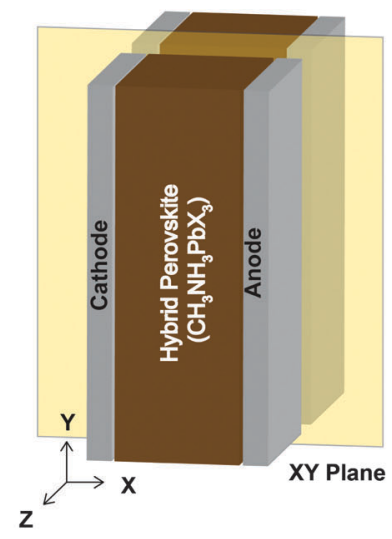

(a)

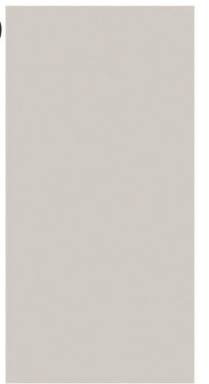

(b)

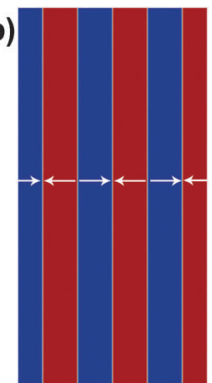

(c)

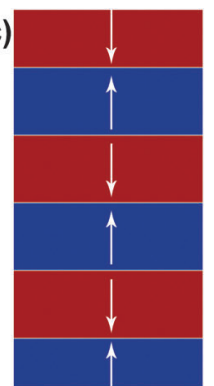

(d)

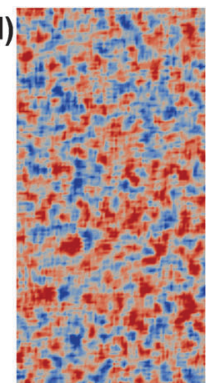

(e)

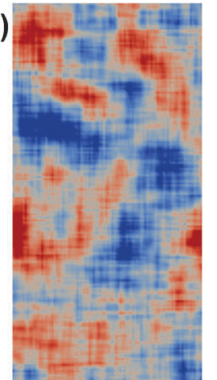

Fig. 3 Cross section of the perovskite solar cell in the $X-Y$ plane showing model ferroelectric domains in the perovskite. The arrows indicate the direction of polarization vector in the respective domains. Assuming high recombination strength, simulations are performed for devices with (a) no polarization, (b) polarization in $\pm X$ direction forming vertical domains, (c) polarization in $\pm Y$ direction forming lateral domains, (d) short order correlated polarization, and (e) long order correlated polarization. The dimensions of all model structures are $300 \mathrm{~nm} \times 600 \mathrm{~nm} \times 600 \mathrm{~nm}$. A number of ten realizations are made for the short and long order correlated microstructures. 


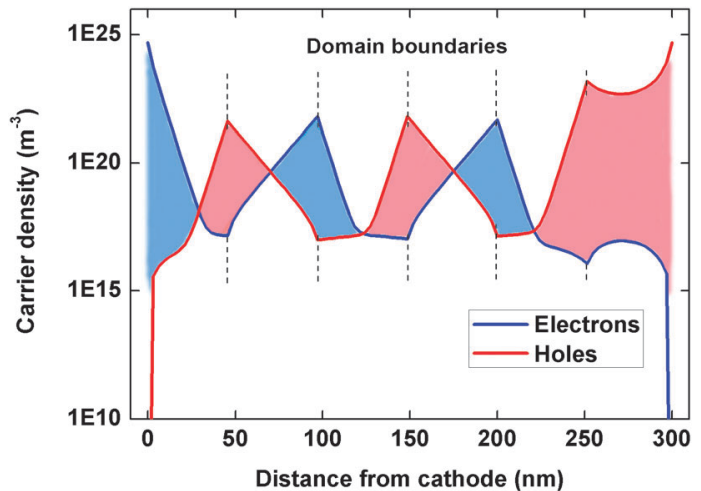

Fig. 5 Carrier concentration profile at short-circuit conditions $\left(V_{\text {applied }}=0 \mathrm{~V}\right)$ along the thickness of the vertical device structure as shown in Fig. 3b. The red and blue colored regions show the segregation of holes and electrons respectively, at the domain boundaries.

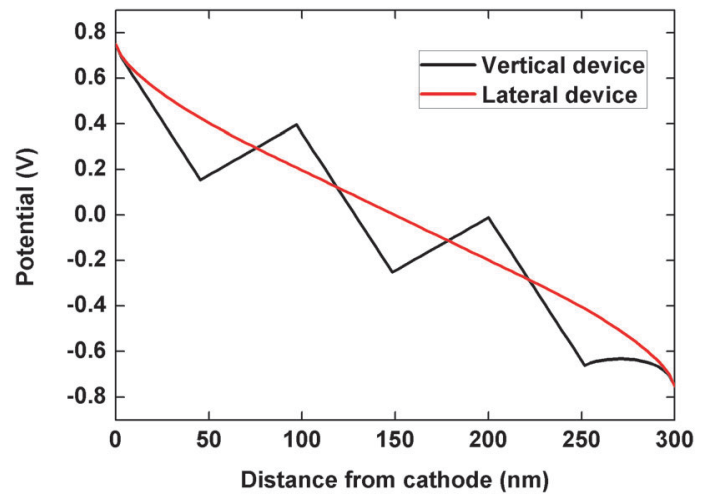

Fig. 6 Potential landscape along the device thickness at short-circuit conditions $\left(V_{\text {applied }}=0 \mathrm{~V}\right)$ for vertical and lateral structures.

boundary) at electrodes as compared to the device with no polarization, which leads to recombination at domain boundaries and an eventual loss in photocurrent. On the other hand, in the lateral device structure, charge carriers do not encounter any domain boundaries during their transport toward the extracting electrodes which explains the high photocurrent. The lateral domain boundaries can therefore be said to induce a channel-like behavior, where the segregated electrons and holes travel along the domain boundaries leading to efficient transport and low recombination loss. The fill factor $(\mathrm{FF})$ which depends on the interplay between the charge transport, extraction and recombination in a device, ${ }^{49}$ is therefore the highest in the case of the lateral structure. Note that, even for small value of polarization density $\left(0.05 \mu \mathrm{C} \mathrm{cm}^{-2}\right)$, the lateral structure shows high $\mathrm{FF}(84.1 \%)$, very close to the calculated idealized limit for $\mathrm{FF} 88 \%$ for a solar cell with $V_{\mathrm{OC}}=0.93 \mathrm{~V}$. For more information on the idealized limit for $\mathrm{FF}$, please refer to the ESI. $\dagger$ A further increase in the polarization density would therefore only cause a marginal increase in $\mathrm{FF}$ as it approaches the idealized limit.

The vertical device structure has been recently studied in the context of inorganic ferroelectric photovoltaics, where the open-circuit voltage was shown to be higher than the band gap of the material. ${ }^{18,19}$ This phenomenon is a result of the high potential created by large number of domains boundaries with a potential step $(\Delta V>10 \mathrm{mV})$ forming a thick device (few micrometers), in addition to the strong polarization of $30-40 \mu \mathrm{C} \mathrm{cm}^{-2}$ in some inorganic ferroelectrics including inorganic perovskites. Their negligible photocurrent ${ }^{18,19}$ can be explained by the strong polarization which produces massive depolarizing fields which forces majority of the charge carriers in the device to recombine. On the other hand, hybrid perovskites films used in PSCs are only a few hundred nanometers thick with fewer domain boundaries, and hybrid perovskites show a low polarization strength of only a few $\mu \mathrm{C} \mathrm{cm}{ }^{-2}$. This results in a weak depolarizing field arising from the bound charges on domain boundaries in hybrid perovskite films, which leads to only small potential step $(\Delta V)$ at domain boundaries and no significant alteration in the potential along the device. Therefore, no significant improvement in the open-circuit voltage is observed in the case of the vertical device structure in PSCs as seen in Fig. 4.

Fig. $7 \mathrm{a}$ and $\mathrm{b}$ show the simulated $J-V$ curves for the lateral and vertical device respectively for variation in the size of domains. To examine the influence of domain size on the device performance of PSCs, we simulate the lateral and vertical device structures with domain sizes ranging from 15 to $100 \mathrm{~nm}$ under the consideration of high recombination strength. Large domains give rise to fewer domain boundaries in the device. In addition, the depolarizing field strength arising from the
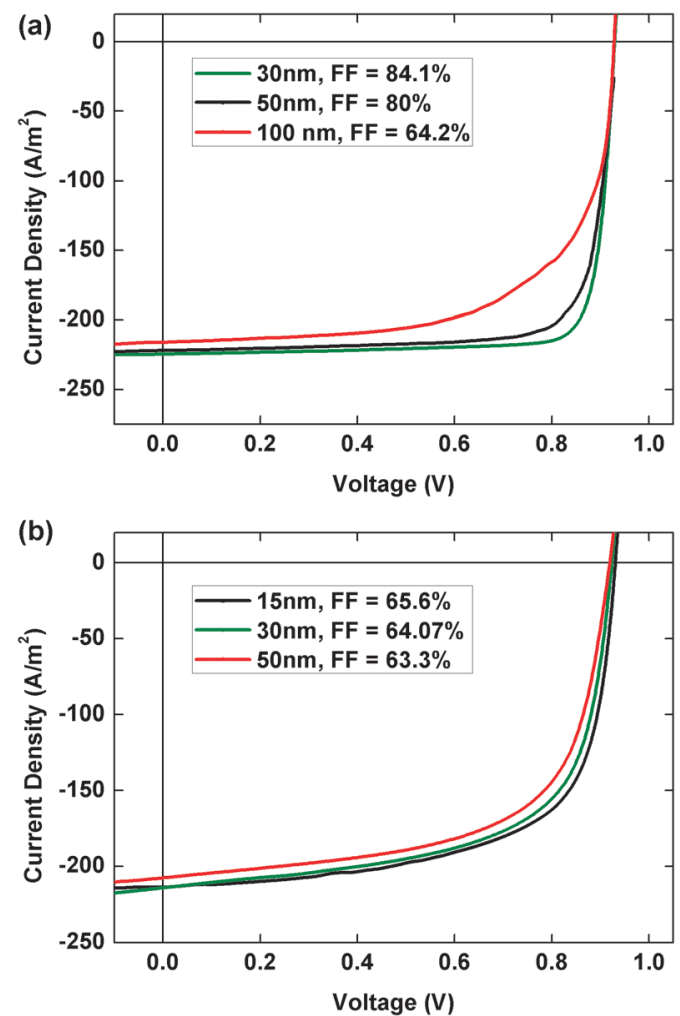

Fig. 7 Simulated J-V curves for (a) lateral and (b) vertical device structures with different domain sizes. 
bound charges on domain boundaries falls inversely with squared distance, according to the Coulomb's law, from the domain boundaries into the domain bulk. This results in lower density of segregated charges at domain boundaries which can be efficiently extracted at the electrodes. Hence, devices with large domain size show poor performance as can be seen in Fig. 7a. In the case of lateral device structure, domain size smaller than $30 \mathrm{~nm}$ shows poor performance (not shown in figure). This is due to the recombination of segregated charges at adjacent domain boundaries, which increases with decrease in the domain size. For the case of vertical device structure, the photocurrent arises from the charge carriers segregated at domain boundaries close to the electrodes and the those charge carriers between the cathode and the first domain boundary, and between the anode and the last domain boundary. Since the decrease in the number of domain boundaries leads to fewer and thicker domains, the change in the photocurrent for the vertical device is not significant as seen from Fig. 7b. Now, if the polarization in the domains were to be strong, as is the case with some inorganic perovskites, the massive depolarizing field would result in recombination of majority of charge carriers at domain boundaries and the photocurrent would only originate from the charges between the electrodes and the first and last domain boundaries. This, once more echoes the observation of low photocurrent in inorganic perovskite devices. Also, as seen in Fig. $7 \mathrm{~b}$, the marginal increase in $V_{\mathrm{OC}}$ with decrease in domain size or increase in number of domain boundaries agrees with the finding by Yang et al. where they observe increase in $V_{\mathrm{OC}}$ with increase in the number of domain boundaries. ${ }^{18}$

The highly ordered polarization domain microstructures shown in Fig. $3 \mathrm{~b}$ and $\mathrm{c}$ are unlikely to occur in actual devices. In a systematic study, Frost et al. modeled the domain behavior by means of Monte-Carlo simulations providing insight into the formation of domain structures in hybrid perovskites. ${ }^{24}$ In order to study similar domain structures, we construct and simulate two complex structures with small $(10 \mathrm{~nm})$ and large $(100 \mathrm{~nm})$ domains defined by short-order and long-order correlated polarization in the perovskite as shown in Fig. 3d and e respectively. Fig. 8a compares the simulated $J-V$ curves for different values of the polarization strength for short-order correlated microstructure. As can be seen, the fill factor (FF) is the parameter which is influenced most significantly with increasing polarization strength. The variation of the FF with polarization density is shown in Fig. $8 \mathrm{~b}$ for both microstructures. As the FF is a directly influenced by the efficiency of charge transport and the degree of recombination in a solar cell, it is evident that the presence of polarization in the perovskite can result in enhanced charge transport and lowering of recombination in a perovskite solar cell (PSC). Since these complex microstructures can be considered to be a composite of the two previously discussed microstructures (Fig. 3b and c), the lateral structure (Fig. 3c) that shows high FF and channel like behavior for charge transport dominates the device operation in these complex microstructures. From Fig. 8b, the FF reaches $83.9 \%$ when $P=0.15 \mu \mathrm{C} \mathrm{cm}^{-2}$ for short-order correlated microstructure and $84.1 \%$ when $P=0.125 \mu \mathrm{C} \mathrm{cm}^{-2}$ for long-order correlated

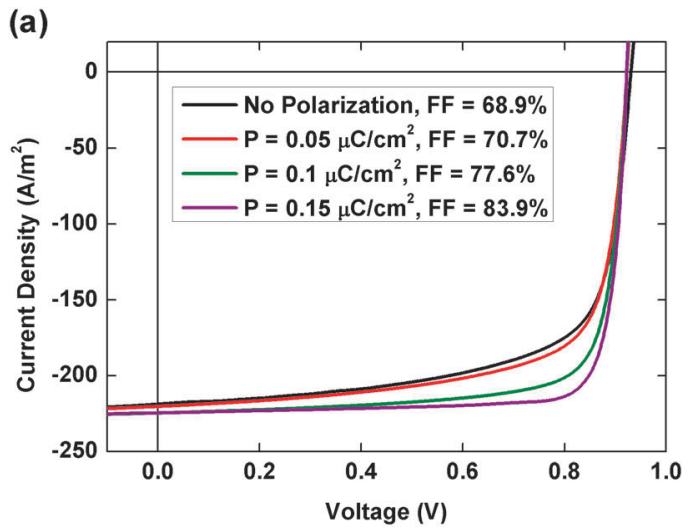

(b)

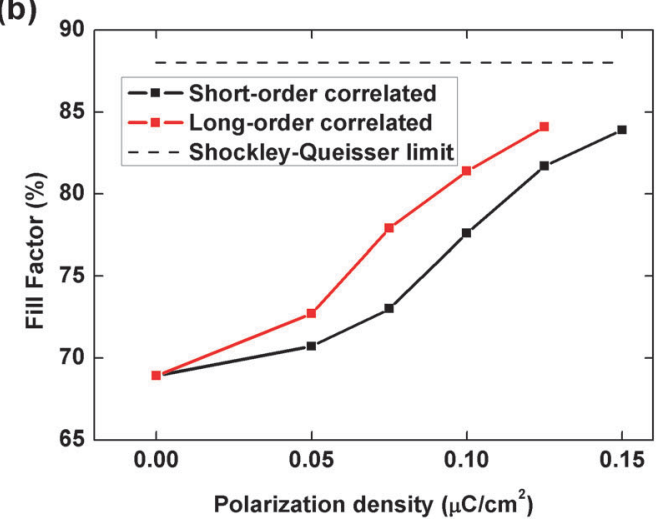

Fig. 8 (a) Simulated $J-V$ curves for the short-order correlated microstructure as a function of polarization density. (b) Variation in fill factor (FF) with polarization density showing the saturation of fill factor with increasing polarization for short-order and long-order correlated microstructures. The dashed line represents the idealized limit for FF (88\%).

microstructure, which is very close to the idealized limit for FF $88 \%$ for a solar cell with $V_{\mathrm{OC}}=0.93 \mathrm{~V}$. Although the $a b$ initio value of polarization in the perovskite is reported to be few $\mu \mathrm{C} \mathrm{cm}^{-2},{ }^{23,48}$ an order of magnitude higher than considered here, a further increase in the value of polarization would lead to a marginal increase in $\mathrm{FF}$.

In the case of vertical device (Fig. 3b), the increase in the polarization strength would lead to increase in the $V_{\mathrm{OC}}$ and decrease in $J_{\mathrm{SC}}$ and $\mathrm{FF}$ as shown in ref. 19. For the case of lateral device (Fig. 3c), increase in polarization strength would only result in a small increase in $\mathrm{FF}$ as the value approaches the idealized limit for FF.

We now consider the scenario of intrinsically low recombination strength in the perovskite by setting the pre-factor for bimolecular recombination, $\gamma_{\text {pre }}=10^{-4}$. This approximate value has been reported by Wehrenfenniget al. in their experimental work. ${ }^{10}$ The comparison between simulated $J-V$ curves for the case with and without polarization in high recombination strength $\left(\gamma_{\text {pre }}=1\right)$ scenario, and no polarization in intrinsically low recombination strength $\left(\gamma_{\text {pre }}=10^{-4}\right)$ scenario, is shown in Fig. 9. Depending on the microstructure, presence of polarization can either result in poor device performance as in the case of vertical domains or can give high fill factor and short-circuit 


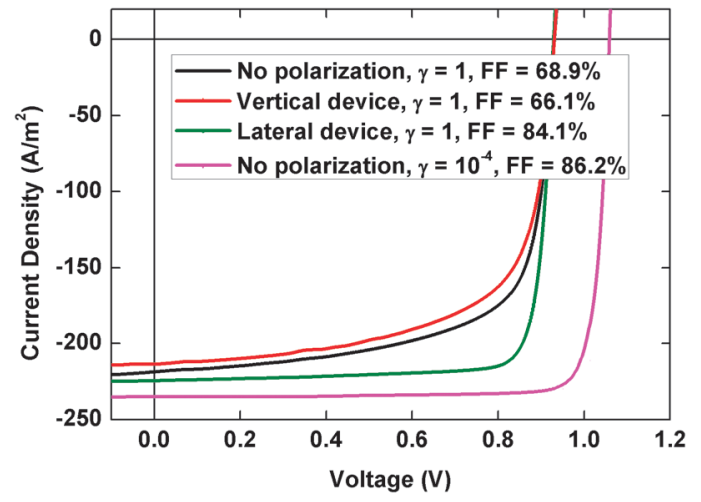

Fig. 9 Simulated $J-V$ curves for various device structures with high recombination strength $\left(\gamma_{\text {pre }}=1\right)$ and low recombination strength $\left(\gamma_{\text {pre }}=10^{-4}\right)$. High $V_{O C}$, which is distinctive of PSCs, can only be explained by the consideration of low recombination strength.

current values as in the case of lateral domains and random correlated structures. However, no device structure is able to explain the high open-circuit voltage $(1.1 \mathrm{~V})$ which is characteristic of high performance PSCs. ${ }^{25,50}$ On the other hand, the device with intrinsically low recombination strength yields a $\mathrm{FF}$ of $86.2 \%, J_{\mathrm{SC}}$ of $235 \mathrm{~A} \mathrm{~m}^{-2}$ and notably, a high $V_{\mathrm{OC}}$ of $1.08 \mathrm{~V}$ comparable to the $V_{\mathrm{OC}}$ of high performance PSCs. This suggests that, the ferroelectric polarization is unlikely to explain the origin of high $V_{\mathrm{OC}}$ of PSCs, and the origin is essentially intrinsic to the perovskite material.

\section{Conclusions}

We studied the influence of mesoscale ferroelectricity in perovskite film on the device performance of the perovskite solar cell, including the short-circuit current $\left(J_{\mathrm{SC}}\right)$, the fill factor $(\mathrm{FF})$, and the open-circuit voltage $\left(V_{\mathrm{OC}}\right)$. We simulated the working of the solar cell with a 3D drift diffusion model and incorporated the mesoscale ferroelectricity in perovskite films by accounting for the bound charge on the polarized domain boundaries. To examine the role of ferroelectricity in the lowering of apparent charge recombination strength in the device, we considered two cases viz. (1) a high recombination strength $\left(\gamma_{\text {pre }}=1\right)$ in presence of ferroelectric polarization scenario, and (2) a low recombination strength $\left(\gamma_{\text {pre }}=10^{-4}\right)$ scenario. In the context of charge recombination, we also investigated the effects that the orientation of the polarization in domains and the size of the domains has on the device performance of the solar cell. We showed that ferroelectric polarization in the perovskite material significantly influences the short-circuit current and fill factor of the solar cell.

We demonstrated that the depolarizing field arising from bound charges is able to effectively separate opposite charge carriers in the bulk of the material resulting in the accumulation of charge carriers at domain boundaries. Polarization along the thickness of the device, which leads to formation of domains parallel to the electrodes, showed poor device performance as electrons and holes buildup at the domain boundaries and eventually recombine to give low short-circuit current and fill factor, with marginal improvement in open-circuit voltage. A device with ordered domains perpendicular to the electrodes and having polarization in the plane of the device showed high $J_{\mathrm{SC}}$ and $\mathrm{FF}$, as electrons and holes encounter no domain boundaries during their transport toward the electrodes. We also examined the influence of the domain size on the device performance, and showed that, for lateral devices the domain size significantly influences charge segregation and recombination dynamics in the device. For vertical devices, owing to the low polarization strength found in hybrid perovskites, the variation in domain size was found to have a marginal influence on the device characteristics.

The presence of highly-ordered polarized domains in the perovskite leads to creation of channels for efficient charge transport and lowering of charge recombination in the device which results into high fill factor (FF) and short-circuit current $\left(J_{\mathrm{SC}}\right)$. Such ordering is however, unlikely to occur in actual devices. In the case of random correlated polarization in domains, a realistic scenario, we showed that electrons and holes appear to follow channels or pathways in the perovskite, giving rise to efficient charge transport and low charge recombination in the solar cell as evidenced by high FFs. Finally, we showed that the high $V_{\text {OC }}$, which is distinctive feature of PSCs, can only be explained by the consideration of the intrinsically low recombination strength $\left(\gamma_{\text {pre }}=10^{-4}\right)$ in hybrid perovskites.

\section{Methods}

\section{Design of device microstructure with short-order and long-order correlated polarization}

We fixed the average value of the polarization, $P_{\text {avg }}$ in the perovskite to $P=0.05,0.075,0.1,0.125,0.15 \mu \mathrm{C} \mathrm{cm}^{-2}$. Initially, the polarization value and direction is set randomly for every grid point in 3D space. Now, a correlation is introduced by smoothing of polarization values at grid points enclosed in a averaging length $\left(r_{x}, r_{y}\right.$ and $\left.r_{z}\right)$ in all three dimensions. The value of the averaging length determines the correlation range in the final structure. The structure shown in Fig. 3d was constructed by setting $r_{x}=r_{y}=r_{z}=2$ grid points, while the one shown in Fig. 3e was constructed by setting $r_{x}=r_{y}=r_{z}=10$ grid points. The smoothed correlated values were then renormalized with $P_{\text {avg }}$ to obtain the final polarization map which defines the microstructures. We made ten realizations for each of the two microstructures.

\section{Acknowledgements}

This is a publication by the FOM Focus Group 'Next Generation Organic Photovoltaics', participating in the Dutch Institute for Fundamental Energy Research (DIFFER). This work is part of the Industrial Partnership Programme (IPP) 'Computational sciences for energy research' of the Foundation for Fundamental Research on Matter (FOM), which is part of the Netherlands Organisation for Scientific Research (NWO). This research programme is co-financed by Shell Global Solutions International B.V. 


\section{References}

1 G. Hodes, Science, 2013, 342, 317-318.

2 M. A. Green, A. Ho-Baillie and H. J. Snaith, Nat. Photonics, 2014, 8, 506-514.

3 N.-G. Park, Mater. Today, 2015, 18, 65-72.

4 H. J. Snaith, J. Phys. Chem. Lett., 2013, 4, 3623-3630.

5 A. Kojima, K. Teshima, Y. Shirai and T. Miyasaka, J. Am. Chem. Soc., 2009, 131, 6050-6051.

6 S. D. Stranks, G. E. Eperon, G. Grancini, C. Menelaou, M. J. Alcocer, T. Leijtens, L. M. Herz, A. Petrozza and H. J. Snaith, Science, 2013, 342, 341-344.

7 E. Edri, S. Kirmayer, S. Mukhopadhyay, K. Gartsman, G. Hodes and D. Cahen, Nat. Commun., 2014, 5, 3461.

8 N. J. Jeon, J. H. Noh, W. S. Yang, Y. C. Kim, S. Ryu, J. Seo and S. I. Seok, Nature, 2015, 517, 476-480.

9 W. S. Yang, J. H. Noh, N. J. Jeon, Y. C. Kim, S. Ryu, J. Seo and S. I. Seok, Science, 2015, 348, 1234-1237.

10 C. Wehrenfennig, G. E. Eperon, M. B. Johnston, H. J. Snaith and L. M. Herz, Adv. Mater., 2014, 26, 1584-1589.

11 J. M. Frost, K. T. Butler, F. Brivio, C. H. Hendon, M. Van Schilfgaarde and A. Walsh, Nano Lett., 2014, 14, 2584-2590.

12 S. Liu, F. Zheng, N. Z. Koocher, H. Takenaka, F. Wang and A. M. Rappe, J. Phys. Chem. Lett., 2015, 6, 693-699.

13 R. E. Cohen, Nature, 1992, 358, 136-138.

14 V. M. Fridkin and B. Popov, Sov. Phys. Usp., 1978, 21, 981.

15 M. Qin, K. Yao and Y. C. Liang, Appl. Phys. Lett., 2008, 93, 122904.

16 S. M. Young and A. M. Rappe, Phys. Rev. Lett., 2012, 109, 116601.

17 T. Choi, S. Lee, Y. Choi, V. Kiryukhin and S.-W. Cheong, Science, 2009, 324, 63-66.

18 S. Yang, J. Seidel, S. Byrnes, P. Shafer, C.-H. Yang, M. Rossell, P. Yu, Y.-H. Chu, J. Scott and J. Ager, et al., Nat. Nanotechnol., 2010, 5, 143-147.

19 J. Seidel, D. Fu, S.-Y. Yang, E. Alarcón-Lladó, J. Wu, R. Ramesh and J. W. Ager III, Phys. Rev. Lett., 2011, 107, 126805.

20 H. Yi, T. Choi, S. Choi, Y. Oh and S.-W. Cheong, Adv. Mater., 2011, 23, 3403-3407.

21 Y. Kutes, L. Ye, Y. Zhou, S. Pang, B. D. Huey and N. P. Padture, J. Phys. Chem. Lett., 2014, 5, 3335-3339.

22 L. Bertoluzzi, R. S. Sanchez, L. Liu, J.-W. Lee, E. Mas-Marza, H. Han, N.-G. Park, I. Mora-Sero and J. Bisquert, Energy Environ. Sci., 2015, 8, 910-915.

23 A. Stroppa, C. Quarti, F. De Angelis and S. Picozzi, J. Phys. Chem. Lett., 2015, 6, 2223-2231.

24 J. M. Frost, K. T. Butler and A. Walsh, APL Mater., 2014, 2, 081506.

25 M. Grätzel, Nat. Mater., 2014, 13, 838-842.

26 J. Guyonnet, Ferroelectric Domain Walls, Springer, Geneva, 2014.

27 C. Momblona, O. Malinkiewicz, C. Roldán-Carmona, A. Soriano, L. Gil-Escrig, E. Bandiello, M. Scheepers, E. Edri and H. Bolink, APL Mater., 2014, 2, 081504.
28 M. Hirasawa, T. Ishihara, T. Goto, K. Uchida and N. Miura, Physica B, 1994, 201, 427-430.

29 P. Gao, M. Grätzel and M. K. Nazeeruddin, Energy Environ. Sci., 2014, 7, 2448-2463.

30 H. Zhou, Q. Chen, G. Li, S. Luo, T.-B. Song, H.-S. Duan, Z. Hong, J. You, Y. Liu and Y. Yang, Science, 2014, 345, 542-546.

31 S. Ryu, J. H. Noh, N. J. Jeon, Y. C. Kim, W. S. Yang, J. Seo and S. I. Seok, Energy Environ. Sci., 2014, 7, 2614-2618.

32 Q.-K. Wang, R.-B. Wang, P.-F. Shen, C. Li, Y.-Q. Li, L.-J. Liu, S. Duhm and J.-X. Tang, Adv. Mater. Interfaces, 2015, 2, 1400528.

33 D. Bi, L. Yang, G. Boschloo, A. Hagfeldt and E. M. Johansson, J. Phys. Chem. Lett., 2013, 4, 1532-1536.

34 S. Aharon, S. Gamliel, B. El Cohen and L. Etgar, Phys. Chem. Chem. Phys., 2014, 16, 10512-10518.

35 A. Dymshits, A. Rotem and L. Etgar, J. Mater. Chem. A, 2014, 2, 20776-20781.

36 S. Gamliel, A. Dymshits, S. Aharon, E. Terkieltaub and L. Etgar, J. Phys. Chem. C, 2015, 119, 19722-19728.

37 D. Bartesaghi and L. J. A. Koster, Adv. Funct. Mater., 2015, 25, 2013-2023.

38 Q. Lin, A. Armin, R. C. R. Nagiri, P. L. Burn and P. Meredith, Nat. Photonics, 2014, 3, 106-112.

39 A. Miyata, A. Mitioglu, P. Plochocka, O. Portugall, J. T.-W. Wang, S. D. Stranks, H. J. Snaith and R. J. Nicholas, Nat. Phys., 2015, 11, 582-587.

40 N. Ashcroft and N. Mermin, Solid State Physics, Saunders College, Philadelphia, 1976.

41 T. Baikie, Y. Fang, J. M. Kadro, M. Schreyer, F. Wei, S. G. Mhaisalkar, M. Graetzel and T. J. White, J. Mater. Chem. A, 2013, 1, 5628-5641.

42 Y. Yamada, T. Nakamura, M. Endo, A. Wakamiya and Y. Kanemitsu, J. Am. Chem. Soc., 2014, 136, 11610-11613.

43 A. Baumann, S. Väth, P. Rieder, M. C. Heiber, K. Tvingstedt and V. Dyakonov, J. Phys. Chem. Lett., 2015, 6, 2350-2354.

44 D. Shi, V. Adinolfi, R. Comin, M. Yuan, E. Alarousu, A. Buin, Y. Chen, S. Hoogland, A. Rothenberger and K. Katsiev, et al., Science, 2015, 347, 519-522.

45 L. J. A. Koster, O. Stenzel, S. D. Oosterhout, M. M. Wienk, V. Schmidt and R. A. Janssen, Adv. Energy Mater., 2013, 3, 615-621.

46 G. Giorgi, J.-I. Fujisawa, H. Segawa and K. Yamashita, J. Phys. Chem. Lett., 2013, 4, 4213-4216.

47 W.-J. Yin, J.-H. Yang, J. Kang, Y. Yan and S.-H. Wei, J. Mater. Chem. A, 2015, 3, 8926-8942.

48 Y. Zhou, F. Huang, Y.-B. Cheng and A. Gray-Weale, Phys. Chem. Chem. Phys., 2015, 17, 22604-22615.

49 D. Bartesaghi, I. del Carmen Pérez, J. Kniepert, S. Roland, M. Turbiez, D. Neher and L. J. A. Koster, Nat. Commun., 2015, 6, 7083.

50 H. S. Jung and N.-G. Park, Small, 2015, 11, 10-25. 